\title{
Canadian Cardiovascular Society Grading Scale Class
}

National Cancer Institute

\section{Source}

National Cancer Institute. Canadian Cardiovascular Society Grading Scale Class. NCI

Thesaurus. Code C66910.

A finding associated with a patient based on the functional classification developed by the Canadian Cardiovascular Society (CCS), for categorizing patients with ang ina. The classification system comprises four classes, which are defined in terms of the level of physical activity associated the with patient's ang inal symptoms. 